Proceedings of the 2010 Winter Simulation Conference

B. Johansson, S. Jain, J. Montoya-Torres, J. Hugan, and E. Yücesan, eds.

\title{
A PARTICIPATIVE MODELLING FRAMEWORK FOR DEVELOPING CONCEPTUAL MODELS IN HEALTHCARE SIMULATION STUDIES
}

\author{
Antuela A. Tako \\ Kathy Kotiadis \\ Operational Research and Management Science \\ Group, Warwick Business School \\ University of Warwick \\ Coventry, CV4 7AL, UNITED KINDGDOM
}

\author{
Christos Vasilakis \\ Clinical Operational Research Unit \\ University College London \\ 4 Taviton Street \\ London, WC1B 0BT, UNITED KINGDOM
}

\begin{abstract}
Conceptual modelling, one of the first stages in a simulation study, is about understanding the situation under study and deciding what and how to model. We argue that stakeholder involvement as part of conceptual modelling could lead to a more successful simulation study with better prospects for implementation. Our work is mainly applied in health care studies, which are characterized by many stakeholders with multiple views and objectives and often a politically charged environment. We develop a participative conceptual modelling framework, which uses tools from soft systems methodology, a problem structuring approach. The benefit of this approach lies in that it supports the conceptual modelling process by engaging stakeholders in a structured and participative way. It involves facilitated workshops, using a set of tools developed. A case study of the conceptual modelling process undertaken for an obesity system is provided to illustrate the proposed framework and tools.
\end{abstract}

\section{INTRODUCTION}

Over the past forty years simulation has been considered a popular decision making tool to support the improvement of healthcare service provision (Jun et al. 1999; Jacobson et al. 2006). The ability to explore alternative scenarios through the use of computer models rather than experimenting with the actual system of interest makes the use of simulation modelling appealing to healthcare practitioners and administrators. Despite the marked increase in healthcare simulation studies over the last forty years, only a few published studies have been reported as successful or had an impact on policy making. The findings of healthcare simulation models rarely find a way towards implementation (Fone et al. 2003).

One of the main reasons leading to incomplete simulation studies (through lack of implementation) is often reported to be the inefficient communication between the simulation experts and the stakeholders (Lehaney and Hlupic 1995). Our work thus, is based on the premise that stakeholder participation in key stages of a simulation study can lead to more successful studies by particularly increasing the chances of implementation of their findings. Indeed since the early years of Operational Research (OR), it has been advocated that "the most fertile tasks are often found by the groups themselves rather than given to them" (Blackett 1950). We believe that conceptual modelling, as one of the first stages in a simulation study, can potentially lend itself to a participative modelling approach.

Conceptual modelling in discrete-event simulation (DES) has been defined as the process of developing a non-software specific description of the computer model, starting from an understanding of the problem situation, to specifying the objectives, inputs, outputs, content, assumptions and simplifications of the model (Robinson 2008a). In this paper conceptual modelling is distinguished from the conceptual 


\section{Tako, Kotiadis and Vasilakis}

model as the process undertaken to reach to the final output, that is the conceptual model. Even though conceptual modelling is considered to be the most important aspect in simulation modelling (Law 2007), at the same time it is thought to be the least understood (Robinson 2006). Hence the need for a framework that guides the modeller during the process of developing a conceptual model has been pointed out (Robinson 2008b).

Developing conceptual models in a participative environment involving stakeholders has received little attention in the DES community. A number of studies attempt to introduce a participative approach to DES modelling (Lehaney and Hlupic 1995; Lehaney and Paul 1996; Lehaney et al. 1999), but they do not explain the process followed for others to reproduce. Furthermore, these studies are not focused around conceptual modelling. The use of a standardised modelling process (framework) and the adoption of facilitation techniques is required to enable a seamless process. In this paper, we present a conceptual modelling framework, called PartiSim for conceptual modelling, which involves the use of a structured and participative modelling process and associated tools as part of the conceptual modelling process. This framework is part of an overall participative simulation modelling approach called PartiSim. Hence the current framework is called PartiSim for conceptual modelling.

The PartiSim framework for conceptual modelling consists of a set of tools and guidelines that enable conceptual modelling to take place as part of two facilitated workshops attended by healthcare professionals. The proposed framework takes forward existing work that suggests the development of conceptual models using problem structuring techniques, and specifically Soft Systems Methodology (SSM) (Kotiadis 2007; Pidd 2007). SSM is considered suitable for use in DES because it undertakes a systemic and systematic approach in understanding a problematic situation (Kotiadis 2007). However, it has not yet been applied to undertake conceptual modelling in a participative way and it cannot be readily adopted in other simulation studies.

The rest of this paper is structured as follows. In the next section the proposed PartiSim framework for conceptual modelling and the associated tools are described. Section 3 provides a case study of an obesity care modelling study, where the PartiSim framework was used to undertake conceptual modelling. We then conclude, discussing the benefits and contribution of the PartiSim framework to conceptual modelling.

\section{THE PARTISIM FRAMEWORK FOR CONCEPTUAL MODELLING}

The PartiSim framework for conceptual modelling involves a number of steps and tools that aid conceptual modelling. It is part of the wider PartiSim approach, which promotes stakeholder participation in different stages of DES modelling studies. PartiSim stands for Participative Simulation Modelling. The PartiSim approach and framework includes six main stages:

1) Initiate the study;

2) Structure the situation of interest;

3) Specify study objectives;

4) Develop simulation model;

5) Experimentation and

6) Implementation.

Four out of the six stages $(2,3,5$ and 6$)$ are undertaken in a participative environment, in the form of facilitated workshops attended by project stakeholders. The stages of the PartiSim framework are based on the typical stages of OR studies (Willemain 1995) as well as the modelling process suggested in simulation textbooks (Pidd 2004; Robinson 2004; Law 2007). The novelty of this framework lies in that it embeds stakeholder participation in specific stages of a simulation study in the form of facilitated workshops. Through facilitation, the modelling team aims to manage stakeholders' expectations and participation. Learning occurs as part of the open discussion where the stakeholder team debate about the situation at hand. A unique characteristic of the PartiSim approach is that the tools and stages suggested 


\section{Tako, Kotiadis and Vasilakis}

can be used in a "pick and mix" format, where according to the situation, time available and the modelling team's familiarity with the situation one chooses to use the most relevant stages or tools.

In this paper we concentrate on the first three stages of the PartiSim framework (Figure 1), which support the development of a conceptual model, hence called PartiSim framework for conceptual modelling. In the next subsections, an overview of the steps involved and the resulting outputs is provided. The framework is then illustrated in section 3, which provides an example of the conceptual modelling process taken in practice, as part of a study of an obesity care system.

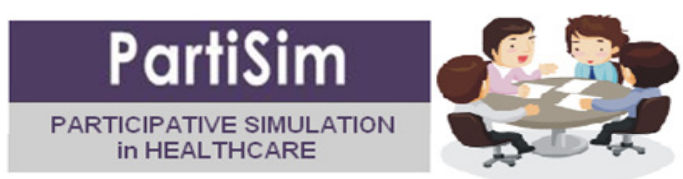

\section{The PartiSim framework for Conceptual Modelling}

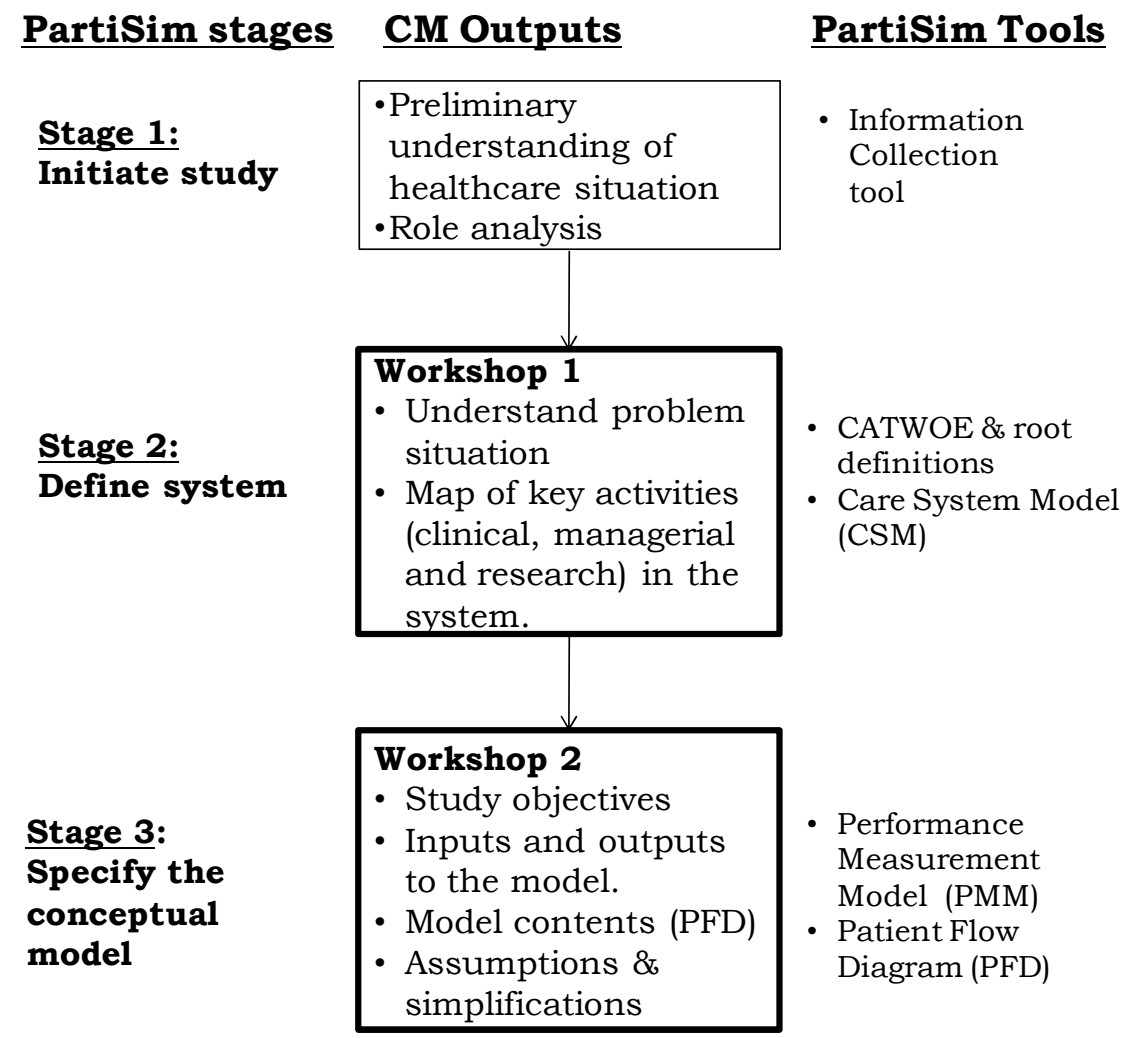

Figure 1: The PartiSim Framework for Conceptual Modelling

\subsection{Stage 1: Initiate the simulation study}

As the title suggests, in the first stage preparatory activities for the simulation study are undertaken. There might not be a specific starting point for this stage, however there will be a vague recognition that a simulation study is required, the simulation modeller or a modelling team is commissioned and a project team is starting to be identified. Two are the main tasks pursued during the initiation stage: 1) gaining a preliminary understanding of the situation of interest and 2) identification of the project team. 


\section{Tako, Kotiadis and Vasilakis}

A preliminary understanding about the situation of interest is mainly achieved by holding informal meetings or one to one interviews with various members of the stakeholder team and on-site observations. Due to healthcare practitioners' limited time availability, it is suggested that meetings with stakeholders are kept to a minimum. A PartiSim Information Collection tool is devised to collect the relevant information at this stage. The information collected involves three main aspects: the problem situation, roles of various people in the system, as well as their opinions about what can be improved.

Setting up a dedicated project team is important in order to ensure a successful start, but also a seamless execution of the study. The members in the project team are distinguished into the stakeholder and the modelling team. The stakeholder team includes a multidisciplinary team of subject matter experts, who have an involvement in the organisation or institution being studied. Ideally no more than about 12 stakeholders should be invited in the facilitated workshops to ensure ease of communication for all participants. Usually a project champion is identified, who can be either someone enthusiastic about the study or the initiator of the study. He/she serves as the link between the modelling team and the stakeholder team. The project champion will play a crucial role throughout the period of the study in motivating other stakeholders and helping to organize workshops. Identifying early on the key stakeholder(s), people with decision making power in the stakeholder organisation is also of importance, to ensure that they are duly involved in the study. Involving key stakeholders in the study enables a higher level of ownership of the simulation study and its results within the organization.

Study initiation is deemed to be a very important stage, not only because key information about the study and the conceptual model is obtained, but also because it sets the basis of the study to be undertaken. Based on the initial information gained, the modelling team can establish the modelling tools and modelling approach suitable to the problem situation, taking also into account the needs expressed by key members of the stakeholder team. The importance of the initiation stage has been also acknowledged in group model building (GMB), a practice developed in system dynamics modelling (Vennix 1999), where conceptualisation takes place and simulation models are built in whole day meetings with the stakeholders. Andersen et al. (1997) call it instead pre-meeting activities.

Based on the information collected at this stage, preliminary models can be also developed, which can be used in the facilitated workshops in the next two stages (these are described in sections 3.2 and 3.3 below). These provide a starting point for the workshops, encouraging discussion and debate. Starting the study from nothing, by jumping straightaway to a workshop, without any preliminary materials, can be time-consuming and unproductive. From his experience in GMB interventions, Vennix (2001) reports that putting in front of the group a preliminary model can stimulate discussion.

\subsection{Stage 2: Define the system (Workshop 1)}

The second stage in the PartiSim framework (Figure 1) focuses on gaining an understanding about the situation of interest and the particular system studied. It takes place in a facilitated workshop environment (Workshop 1) where members of the stakeholder team are invited to participate. This is probably the very first workshop where all PartiSim team members get together in one room. The first workshop can range from 2 hours to half a day. It can be also combined with workshop 2 (section 2.3) in a full day session if it is difficult to get the stakeholders together in two workshops on different days.

The situation of interest is explored utilizing tools adapted from SSM (Checkland 1999). The benefit of using SSM in this stage lies in that it provides useful tools that "make[s] the thinking process coherent and capable of being shared" (Checkland and Scholes 1999, pp. 67). Indeed, Pidd (2007) and Kotiadis and Robinson (2008) suggest that SSM tools can be used to aid conceptual modelling. The former suggests the use of root definitions to support problem understanding, whereas the latter consider developing rich pictures, analyses One, Two and Three (analyses of roles, social system and political system) and purposeful activity models to move from a system description to a conceptual model.

The main activities undertaken in this stage with workshop participants are developing definitions of key aspects about the situation of interest using some of the existing SSM tools (e.g. the CATWOE and root definition) and extended ones (e.g. Care System Model, in short CSM) whilst being guided on the interac- 


\section{Tako, Kotiadis and Vasilakis}

tion by the PartiSim manual on each tool. If the modelling team have prepared preliminary outputs (CATWOE, root definition and CSM) in stage 1, these can be initially used to start the discussions. Stakeholders in this case are invited to revisit and suggest changes/additions.

The CATWOE and root definition are tools used in SSM to define the system studied. The CATWOE tool is used to define the key elements in the system. It is a mnemonic that represents the first letter of each system element (Customers, Actors, Transformation process, World view, Owners and Environmental constraints). For further information on the CATWOE elements the reader is referred to Pidd (2007) and Checkland and Scholes (1999). The CATWOE elements are next combined to define the root definition. The root definition defines the care system of interest in the form of, do X by using Y to achieve Z (Checkland and Scholes 1999). The facilitator ensures that all members of the stakeholder team participate in the discussions. Consensus is aimed so that agreeable (satisficing) and feasible CATWOE and root definition are developed. The CATWOE and root definition set out a broad definition of the system, which leads to a better understanding of the problem situation. These key definitions of the various key aspects of the system are an exercise to focus the stakeholders on the relevant elements of the situation and thus drawing a boundary around the problem. Then they are coerced into expanding the problem situation using the Care System model (CSM).

The Care System Model is an adaptation of the purposeful activity model (PAM) in SSM, to fit specifically situations related to healthcare environments. The CSM tool developed can be used to map out the care system of interest by defining the long- and short-term clinical, managerial and research activities that support that care system. Again a preliminary CSM model already developed by the modelling team in stage 1 can be used to initiate discussions. Obviously, considerations made about the CSM may feed back into the already developed CATWOE and root definition.

The process of developing a CSM consists of collecting the verbs that describe the activities that take place in the care system, structured based on the logical dependencies involved (Rosenhead and Mingers 2001). The activities included in a CSM depend on the situation at hand, although, we believe that three generic activities that take place in health care systems are: clinical, managerial and research. The clinical activities consider the care system from an operational perspective, with regards to the flow of services and/or patients through the care system. The managerial activities refer to the activities required to design and support the management of that system at a strategic level, but it can also include the administrative tasks involved. The research activities consist of activities that support clinical research studies, which often are an additional requirement in health care settings. The research activities involve the design of practices and processes that support the implementation of research and evidence-based projects. Although, research activities were not initially considered as part of the CSM, they were retrospectively included because of their effect on clinical activities in some systems. Indeed, medical research and scientific advancements sit high in the priorities of the majority of healthcare trusts (http://www.nihr.ac.uk). Although the clinical part can be a closer representation to the computer model, depending on the problem situation studied (Kotiadis and Robinson 2008) the research and managerial parts of the CSM will enrich the understanding of the clinical needs leading to a better model. The care system model is further abstracted to develop a more detailed representation of patient flows using the format of process flow diagrams which PartiSim calls patient flow diagram (sub-section 2.3.3).

With the completion of workshop 1, the modelling team reports back the newly agreed outputs to the stakeholder team for reflections. Based on the responses gained the materials are further revised.

\subsection{Stage 3: Specify the conceptual model (Workshop 2)}

In the third stage, a second facilitated workshop with stakeholders takes place, which aims to establish the simulation study objectives and consequently the inputs and outputs to the simulation model, the model contents, and partly data collection, assumptions and simplifications. The relevant information is extracted by using two main PartiSim tools, the performance measurement model (PMM) adapted from 


\section{Tako, Kotiadis and Vasilakis}

(Kotiadis 2007) and the patient flow diagram (PFD). These are explained in the next paragraph and a demonstration of how they were developed in practice is provided in section 3.4.

The Performance Measurement Model (PMM) is used to elicit the simulation study objectives, inputs and outputs by mapping the activities supporting an evaluation of the performance of the system studied. As such, it includes a list of performance measures and activities, which can be grouped into: performance measures, monitoring activities, control activities (activities related to monitoring activities to determine if action is needed - starting with 'determine if') and action to be taken (changes or improvements to the system to achieve the performance measures specified). These are linked in a logical order using arrows. The PMM model developed for the PartiSim framework is based on Kotiadis (2007), but simplified and adapted in a way that it can be used in a participative way in workshops with the stakeholders. The novelty lies in the fact that stakeholders are directly involved in the development of the PMM.

The PMM is developed in a stepwise process. Initially, the three performance criteria (3Es): efficacy, efficiency and effectiveness as identified in SSM interventions (Checkland and Scholes 1999) are considered. Based on the three performance criteria, members are invited to identify the specific performance measures that make sense for judging the performance of the system. Then the performance measurement model diagram is completed or it can be optionally put together after the workshop and sent back to the stakeholders for reflection. The monitoring activities are normally listed first, then the control activities and then action to be taken, based on logical dependencies. It is important that participants agree on the action to be taken before proceeding with the specification of study objectives. Action to be taken represents change(s) that is considered by the group of stakeholders to lead to an improvement. Some of the action to be taken will be evaluated in a DES model and support the formulation of the simulation study objectives.

Establishing the possible change (s) can lead to conflict within the workshop. A voting process is suggested if more than three potential changes are suggested or in the case that reaching consensus is difficult. Based on the objectives agreed the modelling team identifies the inputs and the expected model outputs. Space is provided in the PMM tool to distinguish performance measures into two types: I for Inputs and $\mathrm{O}$ for outputs. With regards to inputs, stakeholders are asked to provide information about the extent that these could vary.

A dedicated tool can be used to define simulation study objectives. As a guide the tool includes the following components: purpose, target performance, change (optional), constraints (Robinson 2004). Objectives can be developed during the workshop or after using the information collected during the workshop. However, after the workshop an iteration of the modelling objectives is recommended, in order to establish the extent of variation of inputs, but also to validate workshop outputs.

Having identified the study objectives, inputs and outputs to the model, the workshop then focuses on establishing model contents. This is achieved by developing a patient flow diagram (PFD), an equivalent communicative model to the process flow diagram in DES. This session serves as a means of attracting the attention of stakeholders to defining more specific steps of the care pathway, with the ultimate aim to decide on the level of detail and content to be included in the simulation model. It also aims to discuss the data inputs required and to discuss issues related to data availability and collection. The communicative model, PFD agreed in this workshop, will be next transformed into a computer model as the study goes on (stage 4 in the PartiSim framework, not covered here as it is not within the scope of this paper).

The PFD is built based on the clinical part of the CSM model. It defines in more detail the possible routes that patients follow in the care system. The majority of health care models represent patients as the entities moving through the system, hence the patient flow diagram is considered to be a reasonable approach to expressing the communicative model. The PFD consists of a sequence of queues i.e. patients queued in a waiting list (represented in circles) and clinical activities (represented in boxes), which form the basis of DES models (and most DES software). They are considered to be a simple and comprehensible diagrammatic tool for participants with little or no knowledge of simulation modelling (Robinson 2004). However, for other models in health care where patients are not the main concern and the PFD may not be necessarily used, the PartiSim approach of facilitated workshops is still applicable. 


\section{Tako, Kotiadis and Vasilakis}

The practice of developing a PFD with and in front of the stakeholder team provides a forum for discussion, where further abstraction takes place and a communicative model is developed based on the stakeholders' shared mental models of the care system, consisting of the flow of activities and the rules involved. The PFD diagram can be drawn on a white board visible by all participants. Obviously various versions and alterations are developed as suggestions and discussions are raised. Hence, ample writing space for different versions of the diagram is necessary. It should be pointed out that a direct connection between the PFD and the clinical part of the CSM can be identified, which in turn enables the ability to move back and forth between the CSM and the PFD.

To ensure full acceptance of the outcomes accrued so far during the workshops, it is suggested that the outputs are fedback to the stakeholder team to comment on. Data collection formally takes place during stage 4 of the PartiSim framework, however, it is important that an agreement is achieved during workshop 2 about data sources and the person responsible.

\section{CASE STUDY: UNDERTAKING CONCEPTUAL MODELLING FOR AN OBESITY MODEL}

This section provides an account of an obesity modelling study undertaken using the PartiSim framework and tools. The study was merely undertaken for the purposes of testing the PartiSim framework for conceptual modelling. However, with time, genuine interest was shown by the stakeholders, which led to a complete PartiSim study.

This particular study was undertaken in two phases. The first two stages of the PartiSim approach were initially undertaken to gain an understanding of the broader system. As a result a Care System Model was agreed with the stakeholders in Workshop 1. At a second phase, the study was resumed with workshop 2, after having established the focus of the study. In this workshop, further abstraction took place, where study efforts concentrated in one particular obesity care unit. By the end of workshop 2, conceptual model specifications were defined and agreed with the stakeholders. The study continued with the next three stages of the overall PartiSim framework, and as a result a DES simulation model of the obesity care service and the pathway followed by its patients was developed. However, these are not within the scope of this paper. We concentrate here on the conceptual modelling process undertaken, stages 1 to 3 . In the next paragraphs, we will describe the process followed and provide some of the conceptual model outputs developed.

\subsection{Overview of the obesity care context}

Obesity poses a major challenge in the USA, UK and worldwide. In the UK, the prevalence of obesity has more than doubled in the last 25 years (Government Office for Science 2007), whereas a quarter of the adult population in England is classified as obese (The NHS Information Centre Lifestyles Statistics 2009). Obesity is a complex issue, affected by a multitude of factors (National Institute for Health and Clinical Excellence 2006). The treatment of obesity from a health care perspective deals with the clinical management of people who are classified as obese based on specific clinical guidelines. In this paper the treatment of obesity refers to the provision of clinical treatment to people who are considered clinically obese or who are referred for specialist care.

The treatment of obesity is increasingly becoming a priority for healthcare services. A number of health care institutions have introduced a number of services aimed at its treatment, including lifestyle treatment, drugs and surgery. The increasing number of obesity levels in the UK has resulted in an increased number of patients seeking access to health care services, where the available capacity and resources cannot always cover the increased demand. Health care institutions providing obesity-related treatment may be at the moment just about managing with the needs, however in the long term, they run the risk of building high waiting lists, with patients spending a long time waiting for the delivery of service. As a result, a number of healthcare institutions (NHS trusts in the UK) risk breaching government directives, such as the 18 week target (patient maximum wait time for treatment) set by the Department of 


\section{Tako, Kotiadis and Vasilakis}

Health in the UK (Department of Health 2004). The current study was undertaken to look into the options of improving the capacity of the obesity care services provided at a London-based hospital.

\subsection{Initiation of the Obesity study}

For the timely completion of the study, the modelling team became aware of the need of maintaining good links with the stakeholder team. Early on during the study role analysis was undertaken and a project champion, representing the health care institution was assigned. One to one meetings were initially held with the project champion and the key stakeholder, in addition to on-site observations of clinics. These activities were kept to a minimum not to obstruct the smooth provision of the care services. However, sufficient information was collected to enable the development of a preliminary CATWOE, root definition and care system model. These were used in workshop 1, to ensure a swift achievement of its objectives. During the study initiation stage, the stakeholder team was also identified, ensuring that a wide representation of different parts of the obesity care system was achieved. The stakeholder team included twelve healthcare professionals (Consultants, Fellows and Nurses) representing a wide range of specialties including, general surgery, chemical biochemistry, anaesthetics, and endocrinology (diabetes and obesity), as well as the Medical Director of the Trust. They were invited to take part in the facilitated workshops. The modelling team involved a team of 3 operational researchers, who took different roles, that of the facilitator, modeller, note keeper etc. throughout the period of the project.

\subsection{Workshop 1- Defining the obesity care system}

In workshop 1, three were the main aspects discussed: the problems faced by the healthcare institution, the CATWOE and root definition and the care system model. Among others, the key issues discussed relevant to a simulation study were the scarcity of resources (doctors, nurses, beds) available for the provision of care services including, out-patient clinics, in-hospital and post operative care. The stakeholder team's concerns were mainly raised due to the continuously increasing patient referrals from GP practices. Hence they were aware of the need for change, which means that they were willing to take action to improve the system. Developing the CATWOE and root definition served as a means of identifying the core purpose of the obesity care system. This set the scene for further developing a care system model of the key activities involved in the system.

The CSM produced in rough format during the workshop listed the clinical, managerial and research activities that support the design and maintenance of the care system in the short- and long-term. Figure 2, is the tidied up version of the CSM which has also been validated by its stakeholders. The CSM diagram is read from top to bottom with the activities at the top preceding the ones at the bottom, which signify the end of the series of activities for that part of the care system. The shaded squares represent an activity which has been further divided into sub-activities.

In the care system model developed for the obesity centre, the clinical activities comprise the possible route that obesity patients (should) take through the care system. The managerial activities include activities required to design and support the management of the system at a strategic level, but it may also include the administrative tasks involved (such as determining available resources or designing new stages in the care system). The research activities were specified in light of the NICE recommendations for research (National Institute for Health and Clinical Excellence 2006) and the hospital's directive to support research along the care pathway. Interrelations can exist between the three groups of activities, hence additional arrows can be added. For example, the managerial activity "Design and set up patient group forum" is connected to the clinical activity "Provide group forum for patients" in Figure 2. 


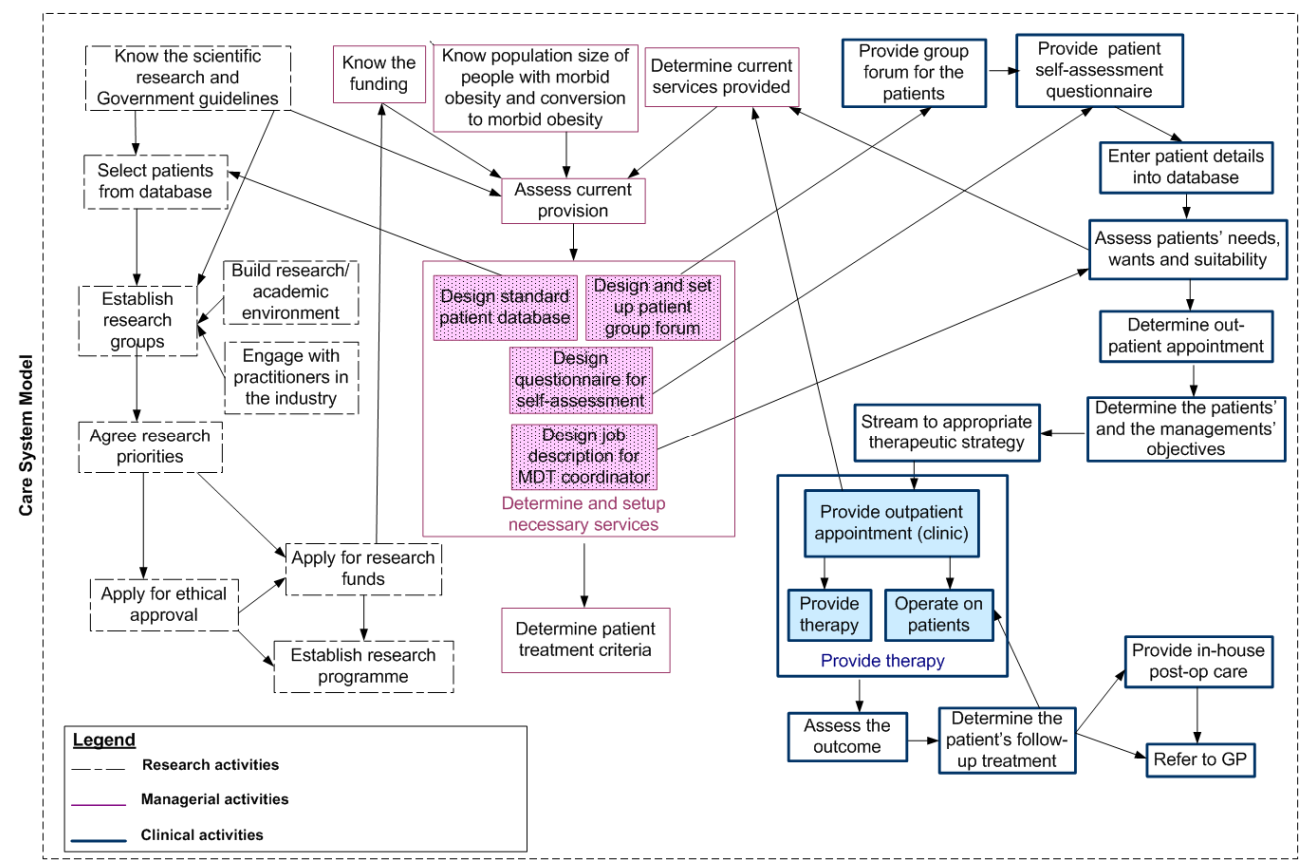

Figure 2: A Care System Model representing the research, managerial and clinical activities in the obesity care system

During the process of designing the CSM, concerns were raised regarding the way the system was run and the problems faced by the health care professionals and patients involved in it. For example some of the issues discussed were the patients repeatedly attending the same clinics, their long waiting times in the system etc. This exercise served as means of bringing out into the open air some of the problems and inefficiencies involved in their obesity system. Especially due to the novel nature of the service, workshop 1 served as a useful exercise to improve the design of the relatively new system and concept (i.e. obesity pathway). New insights were gained by the stakeholders about a number of processes such as patient education for example, which was later on introduced in the real system, where a patient induction session is first provided to the patients before being referred to the appropriate clinic. A general discussion followed as to how to improve the system, but only when getting back into the second workshop, the attention turned on the identification of specific desirable and feasible changes into the obesity care system.

\subsection{Workshop 2 - Specifying the conceptual model for the Obesity system}

In this particular study, the initial obesity care system considered included in reality a wider network of services provided by three separate NHS Trusts. After having developed a wider understanding of the obesity care system, workshop two was next organized with a sub-set of the initial stakeholder team. This team had already designed and implemented the care service as discussed in workshop 1 . Their genuine interest was to identify how a potential increase in capacity and/or decrease in patient referrals (capping the number of patient referrals accepted by the institution) would affect their patient throughput and targets.

The two main themes attended at in workshop 2 were, the development of the performance measurement model (PMM) and the process flow diagram (PFD). In line with the PartiSim philosophy, these were developed in a participative facilitated environment providing brainstorming time for stakeholders to develop their thinking and volunteer their input. The PMM was developed in a stepwise approach. Stakeholders were initially asked to suggest performance measures that should be monitored to ensure that the 3Es are met. The list of monitoring activities identified with the team is provided in Figure 3. Next, rele- 


\section{Tako, Kotiadis and Vasilakis}

vant control (determine if) activities and potential changes to the system were put forward aiming to ensure that the performance criteria were met. A warm up exercise with practical examples (a sort of preliminary model) was initially provided to the stakeholders before interactively developing the real PMM. The PMM in Figure 3 is the final model developed, where activities (boxes) were rearranged after the workshop to improve presentation. For the purpose of identifying the potential inputs and outputs to the system, the performance measures part of the monitoring activities were given the symbol I or O respectively. Later on, during model building, some of the inputs were downgraded into model contents (shown in parenthesis in some of the boxes in Figure 3) depending on the changes that were agreed to explore.

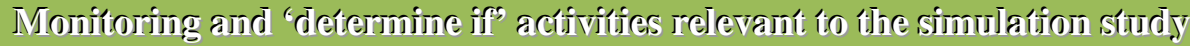

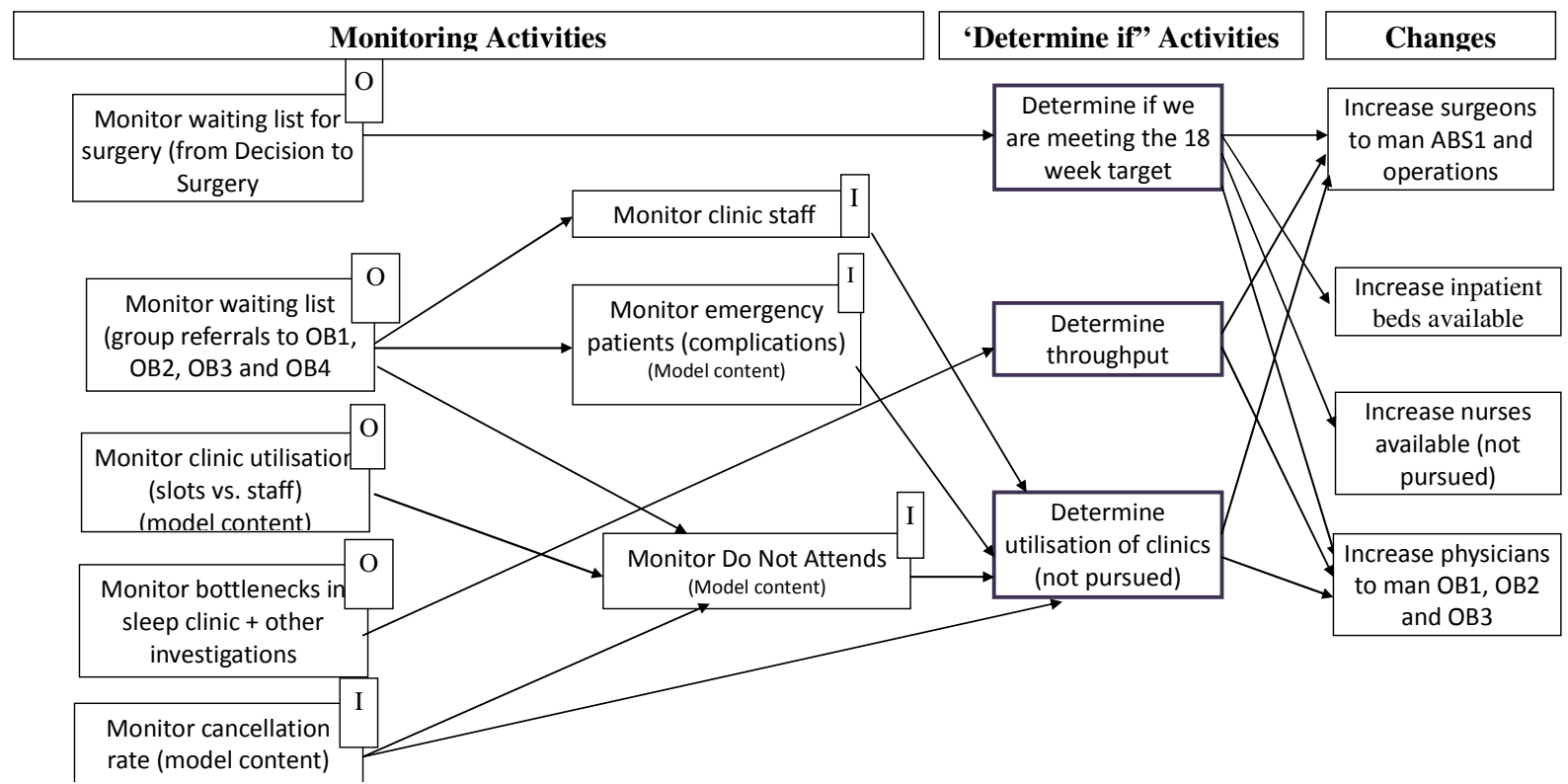

Figure 3: Performance Measurement model developed for the obesity care system.

In this particular study, the process of developing modelling objectives was performed interactively with the stakeholder team. The 'content' for each objective (purpose, target performance, change, constraints) although identified in the workshop was further refined after the workshop because the modelling team required time to format the objectives and the time allocated to this workshop was particularly tight at two hours. A report was put together detailing aspects of the problem to be explored in the study and the simulation study objectives specifying the range over which the changes in the inputs were possible. Dedicated space was provided for the stakeholders to reply/add comments. The final objectives agreed with the stakeholder team were related to identifying the impact that the addition of surgeons, physicians and the reduction of patient referrals to the service would have on the 18-week target. These changes were translated in the form of additional clinics or surgical sessions involving these resources, whereas patient referrals were translated into reduced referral rates. This made possible the identification of the inputs or experimental factors to be entered into the model and the expected outputs/performance measures to be provided by the model.

The next part of the second workshop focused on identifying the model contents. During the workshop this involved mainly identifying the flow that patients follow in the obesity care system. This is equivalent to the clinical part of the CSM (Figure 2), but expanded to include more details, that would be useful for developing the simulation model. From the experience of developing a PFD of the obesity care system in a facilitated workshop, served as a forum for agreement with the stakeholder on which parts of 


\section{Tako, Kotiadis and Vasilakis}

the system were relevant and should be included in the simulation model. After a stage of iterations, the simulation model was developed.

\section{CONCLUSIONS}

This paper described the PartiSim framework for participative conceptual modelling and how it was applied in practice to develop a conceptual model of an obesity care system. In summary, in the first three PartiSim stages, specific aspects of the conceptual model were agreed including: simulation study objectives, inputs and outputs, model contents (PFD). Obviously, data collection as well as identifying the assumptions and simplifications continued during model building, but these were initially partly agreed in Workshop 2. The information relevant to the conceptual model was mainly extracted during stage 3, however the importance of stages 1 and 2 should not be undermined. The information collected in stages 1 and 2 was crucial in gaining an understanding about the problem situation and for making sure that the right problems were tackled (Pidd 2007). A simulation model was built, and its findings were fully accepted by the stakeholder team, when presented at subsequent workshops. As a result of the study implementation took place, mainly in the form of providing additional resources. Furthermore, plans were also being made for building a new operating theatre. Our experience from the obesity study comes to support the premise that stakeholder involvement can indeed lead to success in simulation studies.

The PartiSim framework is a novel approach to modelling that involves stakeholder participation as part of conceptual modelling. While participative modelling has received little attention in the DES community, our experience has proved that it is enormously useful in practice. The benefit of using the PartiSim framework lies in that the relevant information for developing the conceptual model can be collected in a limited period of time, without taking up stakeholders' valuable time. Throughout the process simplified tools and procedures are used for extracting information from stakeholders, with no experience of DES modelling, which enabled them to engage and contribute relevant information to conceptual modelling. The early engagement of stakeholders in the study ensures that stakeholders mould the study to their requirements, but that they also take ownership of the study and its findings. This is crucial for the later stages of the study, especially during the implementation stage. The facilitated workshop environment enables two-way communication and learning to take place. From our experience of undertaking PartiSim studies, the use of a report providing stakeholders a workshop summary and the outputs developed, with dedicated spaces for stakeholders to comment is recommended. The PartiSim approach is currently being tested in other health care systems by the PartiSim founders but others are also encouraged to adopt the approach and reflect their experience from using it.

\section{REFERENCES}

Andersen, D. F., et al. 1997. Group model building: adding more science to the craft. System Dynamics Review 13(2): 187-201.

Blackett, P. M. S. 1950. Operational Research. Operational Research Quarterly (1950-1952) 1(1): 3-6.

Checkland, P. 1999. Systems thinking systems practice. Chichester, Wiley.

Checkland, P. and J. Scholes 1999. Soft Systems Methodology in Action. Chichester, Wiley.

Department of Health 2004. The NHS Improvement Plan: Putting People at the Heart of Public Services. London,

Fone, D., et al. 2003. "Systematic review of the use and value of computer simulation modelling in population health and health care delivery." Journal of Public Health 25(4): 325-335.

Government Office for Science 2007. Foresight. Tackling obesities: future choices.2nd edition, accessed 15 April 2010.

Jacobson, S., et al. 2006. Discrete-Event Simulation of Health Care Systems. Patient Flow: Reducing Delay in Healthcare Delivery. R. W. Hall. New York, Springer: 211-252.

Jun, J. B., et al. 1999. Application of discrete-event simulation in health care clinics: A survey. Journal of the Operational Research Society 50(2): 109-123. 


\section{Tako, Kotiadis and Vasilakis}

Kotiadis, K. 2007. "Using soft systems methodology to determine the simulation study objectives." Journal of Simulation 1: 215-222.

Kotiadis, K. and S. Robinson 2008. Conceptual modelling: Knowledge acquisition and model abstraction. Proceedings of the 2008 Winter Simulation Conference, Miami, Florida, Institute of Electrical and Electronic Engineers, Inc.

Law, A. M. 2007. Simulation modeling and analysis. Boston; London, McGraw-Hill.

Lehaney, B. and V. Hlupic 1995. Simulation modelling for resource allocation and planning in the health sector. Perspectives in Public Health 115(6): 382-385.

National Institute for Health and Clinical Excellence 2006. NICE clinical guideline 43. Obesity: guidance on the prevention, identification, assessment and management of overweight and obesity in adults and children. London, re-issued 29 January 2010.

Pidd, M. 2004. Computer Simulation in Management Science. Chichester, John Wiley \& Sons.

Pidd, M. 2007. Making sure you tackle the right problem: linking hard and soft methods in simulation practice. In Proceedings of the 2007 Winter Simulation Conference, eds. S. G. Henderson, B. Biller, M.-H Hsieh, J. Shortle, J. D. Tew, and R. R. Barton, 1977-1985. Piscataway, New Jersey: Institute of Electrical and Electronics Engineers, Inc.

Robinson, S. 2004. Simulation: The Practice of Model Development and Use. Chichester, John Wiley \& Sons.

Robinson, S. 2006. Conceptual modeling for simulation: issues and research requirements. In Proceedings of the 2006 Winter Simulation Conference. L. F. Perrone, F. P. Wieland, J. Liuet al. Monterey, California, Institute of Electrical and Electronic Engineers, Inc.

Robinson, S. 2008a. Conceptual modelling for simulation Part I: definition and requirements. Journal of the Operational Research Society 59(3): 278-290.

Robinson, S. 2008b. Conceptual modelling for simulation Part II: a framework for conceptual modelling. Journal of the Operational Research Society 59(3): 291-304.

Rosenhead, J. and J. Mingers 2001. Rational Analysis for a problematic world revisited. Chichester, Wiley.

The NHS Information Centre Lifestyles Statistics 2009. Statistics on Obesity, Physical Activity and Diet: England, 2008. 25 February 2009.

Vennix, J. A. M. 1999. Group model-building: tackling messy problems. System Dynamics Review 15(4): 379.

Vennix, J. A. M. 2001. Group Model-building: Facilitating Team Learning Using System Dynamics. Chichester, Wiley.

Willemain, T. R. 1995. "Model Formulation: What Experts Think about and When." Operations Research 43(6): 916-932.

\section{AUTHOR BIOGRAPHIES}

ANTUELA A. TAKO is a Research Fellow at the University of Warwick. She holds a PhD and an MSc in Management Science and Operational Research from the same University. Her research focuses on the simulation model building process, conceptual modelling and comparison of other simulation approaches. Her email address is <antuela.tako@wbs.ac.uk>.

KATHY KOTIADIS is an assistant professor at the Warwick Business School (UK) and co-chair of the UK Simulation Special Interest Group. She holds a BSc and PhD from the University of Kent. Her main research interests include health service modelling and combining problem structuring approaches with DESM. Her email address is <kathy • koatiadis@wbs.ac •uk>. 
CHRISTOS VASILAKIS has an academic background in both operational research and computer science, and several years of experience as a researcher in health systems. Currently a Principal Research Fellow at with the Clinical Operational Research Unit (CORU) at University College London, Christos helps develop practical methods for assisting with real life problems faced by those delivering and managing health services. His email address is $\langle$ c.vasilakis@ucl.ac.uk $\rangle$. 